\title{
Impedance Control for a Free-Floating Robot in the Grasping of a Tumbling Target with Parameter Uncertainty
}

\author{
Satoko Abiko, Roberto Lampariello and Gerd Hirzinger \\ Institute of Robotics and Mechatronics \\ German Aerospace Center (DLR) \\ 82334, Weßling, Germany \\ \{satoko.abiko;roberto.lampariello\}@dlr.de
}

\begin{abstract}
This paper addresses an impedance control for a free-floating space robot in the grasping of a tumbling target with model uncertainty. Firstly, the operational space dynamics for a free-floating robot is derived with a novel, computationally efficient formulation. Then, considering the grasped target as a disturbance force on the end-effector, the proposed control method is completely independent of the target inertial parameters and the end-effector can follow a given trajectory in the presence of model uncertainty. To verify the effectiveness of the proposed method, a three-dimensional realistic numerical simulation is carried out.
\end{abstract}

\section{INTRODUCTION}

Manipulator interaction with the environment requires operational space control schemes. Uncertainties in the environment model give rise to undesired forces on the robot endeffector. In the free-floating dynamics scenario, impacts may cause large deviations in motion and, for inadequate control schemes, high control forces. It is therefore reasonable to avoid impact, if possible, and to introduce compliance at the end-effector.

In this work, the task of grasping a tumbling target by means of a free-floating robot is addressed. The target is assumed to be initially tumbling in some given arbitrary free motion. Following an operational strategy by which the impact between the robot end-effector and the target is minimized at the grasp, the subsequent stabilization motion is analyzed here for the case of uncertainty in the target dynamic model. This leads to a tracking problem, where a given nominal stabilization trajectory has to be tracked, while accounting for the parameter uncertainty.

This problem has been indirectly addressed in the context of adaptive control, in joint space and in operational space [1][2]. A new formulation in the context of impedance control is instead developed here. The resulting method provides a very simple means of deriving the operational space dynamic equations for a free-floating robot as well as a simple control law, based on feedback linearization, for the resolution of the given problem. The control method is tested in simulation for a realistic three-dimensional scenario (see Fig. 1).

The paper is organized as follows. Section II describes some related bibliography. Section III introduces the operational strategy for grasping. In section IV, the dynamic model of a space robot is explained by two approaches. Section $\mathrm{V}$ discusses the theoretical aspects of the control method. In Section VI, the simulation results are illustrated. The conclusions are summarized in Section VII.

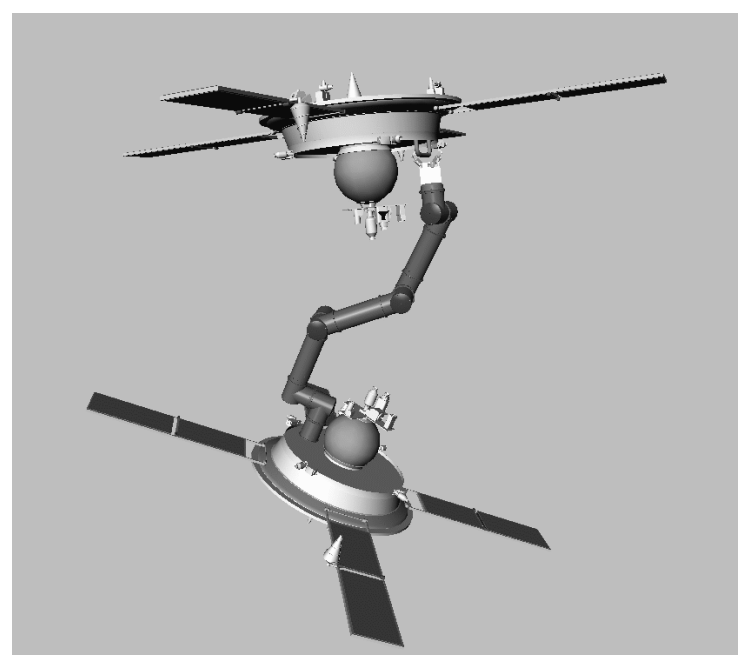

Fig. 1: Chaser-robot and target scenario

\section{RELATED PREVIOUS WORKS}

The space mission to capture a tumbling target by means of a chaser-robot may be divided into four main phases:

(1) following the target motion (Pre-contact),

(2) capturing the target with the manipulator (Contact),

(3) damping out the motion of the target relative to the chaser (Post-contact),

(4) stabilizing the tumbling motion of the compound system (Compound stabilization).

Phases (3) and (4) are usually categorized together in previous research. However, in this paper we consider them separately and focus on phase (3) for motion control with uncertainty in the target dynamic properties.

To cope with the model uncertainty, $\mathrm{Xu}$ and $\mathrm{Gu}$ proposed an adaptive control scheme for space robots in both joint space and operational space [1][2]. Specifically, by considering space robots as under-actuated manipulators, adaptive control in the operational space is introduced in [2]. In [3], both damping out of the chaser-target relative motion and the following compound stabilization are dealt with simultaneously by using the principle of conservation of momentum. However, to carry out both tasks, we need to choose a proper trajectory carefully.

With regards to phase (2), some previous research has analyzed the impact between the target and the manipulator 
[4][5][6]. Nenchev et. al. discussed the effect of the impact and proposed an attitude control scheme for the satellite, based on the reaction null space for post-contact [4]. In [5], the dynamic equations in the operational space are introduced and the configuration to minimize the impact is discussed in the planar case. In [6], Wee also analyzed the dynamical contact between two bodies and revealed that the impulse at contact can be minimized by optimal trajectory planing. However, these studies do not consider the capturing of the target, namely the model uncertainty in its dynamics.

\section{SCENARIO AND ASSUMPTIONS IN THE GRASPING OF A TUMBLING TARGET}

In our envisioned scenario, it is assumed that an inverse kinematics algorithm provides the ideal robot trajectory to align the end-effector velocity with that of the grasping point on the target, such that the impact at the grasp is minimal. This may be achieved by means of an ideal visual path tracker. Subsequently, a motion planner provides a stabilization trajectory to bring the relative motion between the robot and the target to zero, while ensuring feasibility of the task. However, due to parameter uncertainty in the target, this trajectory will require feedback tracking control.

The tracking problem of the stabilization trajectory may be formulated in joint or in operational space. It is however important to realize that the target parameter uncertainty may be treated as an adaptive control problem [1], if the target dynamics wants to be included into the control formulation. This however, does not allow to formulate the more general impedance control theory, which should be independent of the target dynamics, to include consideration of impact forces. In the problem addressed here, the target uncertainty is seen as a disturbance force on the end-effector, with respect to the expected internal force involved in the stabilizing motion.

\section{MODELING AND EQUATIONS OF MOTION}

This section introduces the model of a space robot. As described above, the focus of this research is to follow a desired trajectory in operational space when the space robot grasps the target with unknown dynamic properties. Since our interest is the influence of the contact forces on the environment, or on the target motion in relation to the chaser, it is convenient to refer to operational space schemes.

To derive the dynamics of free-floating space robots in the operational space, two approaches are possible. The dynamic equations of free-floating space robots are generally expressed with linear and angular velocities of the base and the motion rate of each joint as generalized coordinates[7]. However, considering the system switched around, modeled from the end-effector to the base, it can be represented by the motion of the end effector and that of the joints in the same structure as in the conventional expression. This scheme is termed here the inverted chain approach. On the other hand, we can also obtain the dynamics in the operational space from the conventional representation of space robots as introduced in [5] in a straightforward manner, which we term here the forward chain approach.

The following subsections explain the dynamic equations of the system, developed in the two ways mentioned above, for a serial rigid-link manipulator attached to a floating base, as shown in Fig. 2. It is shown that the inverted chain

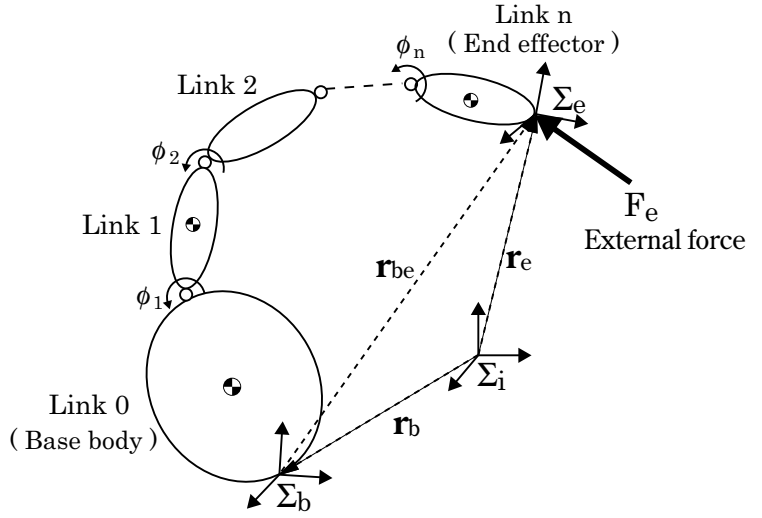

Fig. 2: General model for a space robot with external force

approach has a computational advantage with respect to the forward chain approach.

\section{A. Dynamics for space robots - Inverted chain approach}

Differing from a ground based manipulator, the model of a free-floating space robot is invertible from the end-effector to the base, in the sense of no fixed base of the system. This subsection explains the dynamics of a space robot in this sense.

1) Equation of motion: Considering the generalized coordinates as the linear and angular velocities of the end-effector, $\dot{\boldsymbol{x}}_{e}=\left(\boldsymbol{v}_{e}^{T}, \boldsymbol{\omega}_{e}^{T}\right)^{T} \in R^{6 \times 1}$, and the motion rate of the joints, $\dot{\phi} \in R^{n \times 1}$, the equations of motion are expressed in the following form:

$$
\begin{aligned}
{\left[\begin{array}{cc}
\boldsymbol{H}_{e} & \boldsymbol{H}_{e m} \\
\boldsymbol{H}_{e m}^{T} & \boldsymbol{H}_{m}
\end{array}\right]\left[\begin{array}{c}
\ddot{\boldsymbol{x}}_{e} \\
\ddot{\boldsymbol{\phi}}
\end{array}\right] } & +\left[\begin{array}{c}
\boldsymbol{c}_{e} \\
\boldsymbol{c}_{m}
\end{array}\right] \\
& =\left[\begin{array}{c}
\mathcal{F}_{e} \\
\boldsymbol{\tau}
\end{array}\right]+\left[\begin{array}{c}
\boldsymbol{J}_{e}^{T} \\
\boldsymbol{J}_{m}^{T}
\end{array}\right] \mathcal{F}_{b}
\end{aligned}
$$

The symbols used here are defined as follows:

$$
\begin{array}{llll}
\boldsymbol{H}_{e} & \in R^{6 \times 6} & : & \text { inertia matrix on the end-effector. } \\
\boldsymbol{H}_{m} & \in R^{n \times n} & : & \text { inertia matrix of the arm. } \\
\boldsymbol{H}_{e m} & \in R^{6 \times n} & : & \begin{array}{l}
\text { coupling inertia matrix between the } \\
\text { end-effector and the arm. }
\end{array} \\
\boldsymbol{c}_{e} & \in R^{6 \times 1} & : & \begin{array}{l}
\text { non-linear velocity dependent term } \\
\text { on the end-effector. }
\end{array} \\
\boldsymbol{c}_{m} & \in R^{n \times 1} & : \begin{array}{l}
\text { non-linear velocity dependent term } \\
\text { of the arm. }
\end{array} \\
\mathcal{F}_{e} & \in R^{6 \times 1} \quad: \quad \begin{array}{l}
\text { force and moment exerted on the } \\
\text { end-effector. }
\end{array} \\
\mathcal{F}_{b} & \in R^{n \times 1} & : \begin{array}{l}
\text { force and moment exerted on the } \\
\text { base. }
\end{array} \\
\boldsymbol{\tau} & \in R^{n \times 1} & : & \text { torque on the joints. }
\end{array}
$$

In the case that $\mathcal{F}_{b}$ is generated actively (e.g. jet thrusters or reaction wheels etc.), the system is called a free-flying 
robot. On the other hand, if no active actuators are applied on the base, the system is termed a free-floating robot. In this paper, we consider the free-floating robot.

By canceling out the end-effector acceleration $\ddot{\boldsymbol{x}}_{e}$ in (1), the equations of motion can be reduced to the joint space formulation:

$$
\widehat{\boldsymbol{H}}_{m} \ddot{\boldsymbol{\phi}}+\widehat{\boldsymbol{c}}_{m}=\boldsymbol{\tau}-\boldsymbol{H}_{e m}^{T} \boldsymbol{H}_{e}^{-1} \mathcal{F}_{e}+\widehat{\boldsymbol{J}}_{m} \mathcal{F}_{b}
$$

where $\widehat{\boldsymbol{H}}_{m}=\boldsymbol{H}_{m}-\boldsymbol{H}_{e m}^{T} \boldsymbol{H}_{e}^{-1} \boldsymbol{H}_{e m}, \widehat{\boldsymbol{c}}_{m}=\boldsymbol{c}_{m}-$ $\boldsymbol{H}_{e m}^{T} \boldsymbol{H}_{e}^{-1} \boldsymbol{c}_{e}$, and $\widehat{\boldsymbol{J}}_{m}=\boldsymbol{J}_{m}-\boldsymbol{J}_{e} \boldsymbol{H}_{e}^{-1} \boldsymbol{H}_{e m}$.

2) Equations of motion in the operational space: The upper part of (1) clearly describes the equations of motion in the operational space:

$$
\boldsymbol{H}_{e} \ddot{x}_{e}+\boldsymbol{c}_{e}=-\boldsymbol{H}_{e m} \ddot{\boldsymbol{\phi}}+\mathcal{F}_{e}+\boldsymbol{J}_{e}^{T} \mathcal{F}_{b}
$$

where $\mathcal{F}_{i}=-\boldsymbol{H}_{\text {em }} \ddot{\boldsymbol{\phi}}$ describes the input command generated by the joint actuators.

Substituting equation (2) into (3), the acceleration of the end-effector can be represented by the torques on the joints and by the external forces/torques on the end-effector, with the assumption of no external forces/torques on the base $\mathcal{F}_{b}=\mathbf{0}$ :

$$
\begin{aligned}
\ddot{\boldsymbol{x}}_{e} & =-\boldsymbol{H}_{e}^{-1} \boldsymbol{H}_{e m} \widehat{\boldsymbol{H}}_{m}^{-1} \boldsymbol{\tau} \\
& +\boldsymbol{H}_{e}^{-1}\left(\boldsymbol{I}+\boldsymbol{H}_{e m} \widehat{\boldsymbol{H}}_{m}^{-1} \boldsymbol{H}_{e m}^{T} \boldsymbol{H}_{e}^{-1}\right) \mathcal{F}_{e}+\boldsymbol{d}
\end{aligned}
$$

where $\boldsymbol{d}=-\boldsymbol{H}_{e}^{-1} \boldsymbol{c}_{e}+\boldsymbol{H}_{e}^{-1} \boldsymbol{H}_{e m} \widehat{\boldsymbol{H}}_{m}^{-1} \widehat{\boldsymbol{c}}_{m}$ denotes the Coriolis and centrifugal forces in operational space.

\section{B. Dynamics for space robots - Forward chain approach}

The other way to derive the equations of motion in the operational space is by applying the classical expressions to a space robot. A similar approach has been introduced in [5]. To formulate the dynamics of the system with the forward chain approach, the general expressions for space robots are briefly reviewed first.

1) Equations of motion: The equations of motion in joint space for a free-floating space robot are generally expressed in the following form [7]:

$$
\boldsymbol{H}_{m}^{*} \ddot{\boldsymbol{\phi}}+\boldsymbol{c}_{m}^{*}=\boldsymbol{\tau}+\boldsymbol{J}_{m}^{* T} \mathcal{F}_{e}
$$

where $\boldsymbol{H}_{m}^{*} \in R^{6 \times 6}, \boldsymbol{c}_{m}^{*} \in R^{6 \times 1}$ and $\boldsymbol{J}_{m}^{*} \in R^{6 \times n}$ are the so-called generalized inertia matrix, generalized Coriolis and centrifugal forces and generalized Jacobian of a space robot, respectively [4].

2) Linear and Angular Momentum Equations: The motion of the system is also governed by the momentum equations in the absence of external forces:

$$
\left[\begin{array}{l}
\mathcal{P}_{0} \\
\mathcal{L}_{0}
\end{array}\right]=\boldsymbol{H}_{b} \dot{\boldsymbol{x}}_{b}+\boldsymbol{H}_{b m} \dot{\boldsymbol{\phi}}
$$

in which the generalized coordinates are the linear and angular velocities of the base satellite, $\dot{x}_{b}=\left(\boldsymbol{v}_{b}^{T}, \omega_{b}^{T}\right)^{T} \in R^{6 \times 1}$ and the motion rate of the joints, $\dot{\phi} \in R^{n \times 1}$. $\boldsymbol{H}_{b} \in R^{6 \times 6}$ and $\boldsymbol{H}_{b m} \in R^{6 \times n}$ denote the inertia matrix of the base and the coupling inertia between the base and the joints, respectively. Note that $\mathcal{P}_{0}$ and $\mathcal{L}_{0}$ represent the total linear and angular momentum around the base coordinate system.
The total linear and angular momentum can be also expressed around the end-effector coordinates:

$$
\left[\begin{array}{l}
\mathcal{P} \\
\mathcal{L}
\end{array}\right]=\boldsymbol{H}_{e} \dot{\boldsymbol{x}}_{e}+\boldsymbol{H}_{\text {em }} \dot{\boldsymbol{\phi}}
$$

The expression of the angular momentum is generally dependent on the reference frame. Therefore, the following transformation can be obtained between (6) and (7):

$$
\begin{aligned}
{\left[\begin{array}{c}
\mathcal{P}_{0} \\
\mathcal{L}_{0}
\end{array}\right] } & =\left[\begin{array}{cc}
\boldsymbol{E}_{3} & \mathbf{0}_{3} \\
\boldsymbol{r}_{b e} \times & \boldsymbol{E}_{3}
\end{array}\right]\left[\begin{array}{c}
\mathcal{P} \\
\mathcal{L}
\end{array}\right] \\
& =\left[\begin{array}{cc}
\boldsymbol{E}_{3} & -\boldsymbol{r}_{b e} \times \\
\mathbf{0}_{3} & \boldsymbol{E}_{3}
\end{array}\right]^{T}\left[\begin{array}{c}
\mathcal{P} \\
\mathcal{L}
\end{array}\right] \\
& =\boldsymbol{J}_{b}^{T}\left[\begin{array}{c}
\mathcal{P} \\
\mathcal{L}
\end{array}\right]
\end{aligned}
$$

where $\boldsymbol{E}_{3} \in R^{3 \times 3}$ and $\mathbf{0}_{3} \in R^{3 \times 3}$ respectively denote the identity matrix and the zero matrix. $\boldsymbol{r}_{b e}$ represents the vector from the base to the end effector and the operator $\{x\}$ indicates the cross product. $\boldsymbol{J}_{b}$ denotes the Jacobian matrix from the base to the end effector.

3) Kinematics: The kinematic relationship between the operational and the joint space is described as follows, by using (8) and the generalized Jacobian matrix $\boldsymbol{J}_{m}^{*}$ which include the dynamic parameters of the system:

$$
\begin{aligned}
\dot{\boldsymbol{x}}_{e} & =\boldsymbol{J}_{m}^{*} \dot{\phi}+\boldsymbol{J}_{b} \boldsymbol{H}_{b}^{-1}\left[\begin{array}{l}
\mathcal{P}_{0} \\
\mathcal{L}_{0}
\end{array}\right] \\
& =\boldsymbol{J}_{m}^{*} \dot{\phi}+\boldsymbol{\Lambda}_{b}^{-1}\left[\begin{array}{c}
\mathcal{P} \\
\mathcal{L}
\end{array}\right]
\end{aligned}
$$

where $\boldsymbol{\Lambda}_{b}=\left(\boldsymbol{J}_{b} \boldsymbol{H}_{b}^{-1} \boldsymbol{J}_{b}^{T}\right)^{-1} \in R^{6 \times 6}$ denotes the inertia matrix of the base projected on the end-effector.

4) Equations of motion in the operational space: By using equation (5) and (9), the dynamic equations in the operational space can be obtained in a straightforward manner.

The derivative of (9) leads to the equations of motion in the operational space in function of the acceleration of the end-effector and that of the joints:

$$
\ddot{\boldsymbol{x}}_{e}=\boldsymbol{J}_{m}^{*} \ddot{\boldsymbol{\phi}}+\boldsymbol{\Lambda}_{b}^{-1} \mathcal{F}_{e}+\dot{\boldsymbol{J}}_{m}^{*} \dot{\boldsymbol{\phi}}+\dot{\boldsymbol{\Lambda}}_{b}^{-1}\left[\begin{array}{l}
\mathcal{P} \\
\mathcal{L}
\end{array}\right]
$$

To obtain the equations of motion in function of the acceleration of the end effector and the torques on each joint, equation (5) is substituted into equation (10).

$$
\ddot{\boldsymbol{x}}_{e}=\boldsymbol{J}_{m}^{*} \boldsymbol{H}_{m}^{*-1} \boldsymbol{\tau}+\left(\boldsymbol{\Lambda}^{-1}+\boldsymbol{\Lambda}_{b}^{-1}\right) \mathcal{F}_{e}+\boldsymbol{\mu}
$$

where $\boldsymbol{\Lambda}=\left(\boldsymbol{J}_{m}^{*} \boldsymbol{H}_{m}^{*-1} \boldsymbol{J}_{m}^{* T}\right)^{-1} \in R^{6 \times 6}$ denotes the kinetic energy in the operational space for ground based manipulators[8][9]. $\left(\boldsymbol{\Lambda}^{-1}+\boldsymbol{\Lambda}_{b}^{-1}\right)$ is named the extended generalized inertia tensor [5]. $\boldsymbol{\mu}=-\boldsymbol{J}_{m}^{*} \boldsymbol{H}_{m}^{*-1} \boldsymbol{c}_{m}^{*}+\dot{\boldsymbol{J}}_{m}^{*} \dot{\boldsymbol{\phi}}+$ $\frac{d}{d t}\left(\boldsymbol{\Lambda}_{b}^{-1}\right)\left[\begin{array}{c}\mathcal{P} \\ \mathcal{L}\end{array}\right] \in R^{6 \times 1}$ expresses the Coriolis and centrifugal forces in operational space. 


\section{Comparison between the two expressions}

Let us compare the above two expressions in the two previous subsections for the operational space dynamics. By comparing (3) and (10), or (4) and (11), it is clear that the following equalities result.

$$
\begin{aligned}
\widehat{H}_{e} & =\boldsymbol{\Lambda}_{b} \\
\widehat{\boldsymbol{H}}_{m} & =\boldsymbol{H}_{m}^{*} \\
-\boldsymbol{H}_{e}^{-1} \boldsymbol{H}_{e m} & =\boldsymbol{J}_{m}^{*} \\
-\boldsymbol{H}_{e}^{-1} \boldsymbol{c}_{e} & =\dot{\boldsymbol{J}}^{*} \dot{\phi}+\frac{d}{d t}\left(\boldsymbol{\Lambda}_{b}^{-1}\right)\left[\begin{array}{c}
\mathcal{P} \\
\mathcal{L}
\end{array}\right]
\end{aligned}
$$

The above relationships are obtained by comparison of the single terms. Therefore, the two equations (3) and (10), or (4) and (11) are equivalent. However, expression (3) has an advantage over expression (10) in view of the computational consumption ( e.g. the calculation of $\boldsymbol{\Lambda}=\left(\boldsymbol{J}_{m}^{*} \boldsymbol{H}_{m}^{*-1} \boldsymbol{J}_{m}^{* T}\right)^{-1}$ and $\left.\boldsymbol{\Lambda}_{b}=\left(\boldsymbol{J}_{b} \boldsymbol{H}_{b}^{-1} \boldsymbol{J}_{b}^{T}\right)^{-1}\right)$. In case of limited computational performance, it is desired that the computational consuming is reduced as much as possible. By using the inverted chain approach, we can calculate the inertia matrix of the endeffector $\boldsymbol{H}_{e}$ directly instead of with the calculation of $\boldsymbol{\Lambda}_{b}=$ $\left(\boldsymbol{J}_{b} \boldsymbol{H}_{b}^{-1} \boldsymbol{J}_{b}^{T}\right)^{-1}$, which involves two matrix inversions.

\section{IMPEDANCE CONTROL IN THE OPERATIONAL SPACE}

As mentioned in section III, the robot should follow a given trajectory to dampen out the motion of the end-effector with respect to the base coordinate fame, to eventually stabilize the system.

This section proposes an interaction control for space robots, which is based on the ideas of impedance control for ground based manipulators [10]. However, the interaction control is exploited here to follow a desired trajectory, by regarding the internal forces at the grasping point of the target as external forces on the end-effector.

In the grasping of the tumbling target, the uncertainty in the target inertial properties may give rise to unsustainable forces at the end-effector. The mechanical impedance (massdamper-spring) imposed on the end-effector, is then useful to avoid this situation. The advantage of the impedance control is that the inertia (mass) characteristics of the end effector can be suitably designed. For example, if the impedance characteristics of the end-effector are made similar to those of the target, this leads to the so-called mechanical impedance matching [11]. As such, impact can be treated together with uncertainty.

Furthermore, he impedance control is developed in the operational space, since the impedance characteristics should be determined with respect to the environment, or to the target motion, or even to account for collision avoidance for the chaser-target compound.

For the motion damping, let us consider a control model which consists of the space robot alone, while the model for the real system includes the target with some model uncertainty, as shown in Fig. 3. The latter uncertainty is source of error in the motion planning solution for the desired damping trajectory $\boldsymbol{x}_{e}^{d}$. The internal forces between the endeffector and the target can be expressed in function of the known motion of the system, with the assumption of no external forces. Therefore, these can then be modeled as

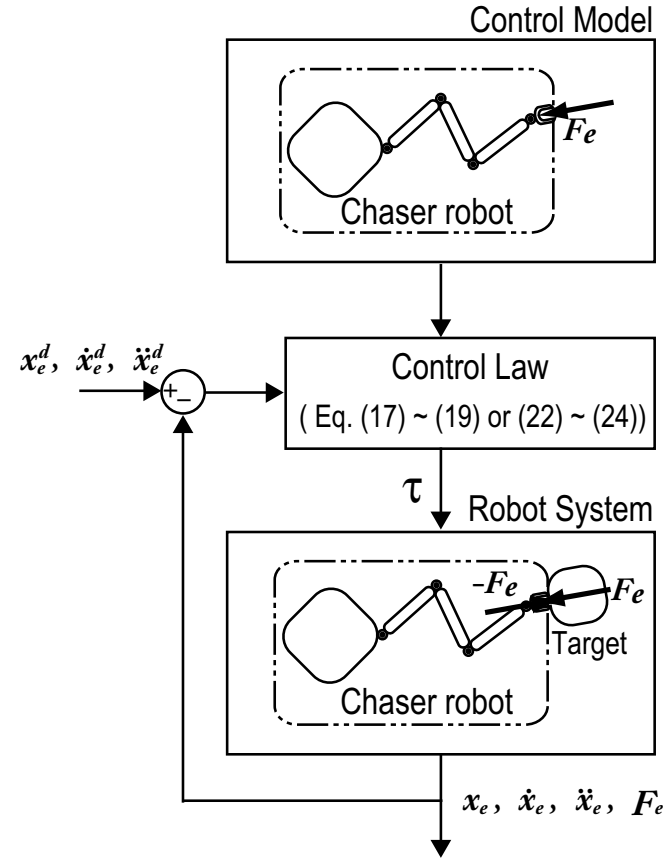

Fig. 3: Control block diagram

virtual external forces for the control model, as shown in Fig. 3.

In the case without force measurement, the control law can be determined as follows (c.f. (3)):

$$
\mathcal{F}_{i}=-\boldsymbol{H}_{e m} \ddot{\boldsymbol{\phi}}=\boldsymbol{H}_{e} \boldsymbol{y}+\boldsymbol{c}_{e}
$$

with

$$
\boldsymbol{y}=\ddot{\boldsymbol{x}}_{e}^{d}-\boldsymbol{\Lambda}_{d}^{-1}\left(\boldsymbol{D}_{d} \dot{\widetilde{x}}_{e}+\boldsymbol{K}_{d} \widetilde{x}_{e}\right)
$$

where $\widetilde{\boldsymbol{x}}_{e}=\boldsymbol{x}_{e}-\boldsymbol{x}_{e}^{d}$ denotes the error between the operational space position and orientation $\boldsymbol{x}_{e}$ and the desired equilibrium point $\boldsymbol{x}_{e}^{d} \cdot \boldsymbol{\Lambda}_{d}, \boldsymbol{D}_{d}$ and $\boldsymbol{K}_{d}$, respectively, represent the desired inertia, damping and stiffness matrices which specify the dynamic impedance behavior of the end-effector. The input command on torque level is obtained from (2):

$$
\boldsymbol{\tau}=-\widehat{\boldsymbol{H}}_{m} \boldsymbol{H}_{e m}^{+} \mathcal{F}_{i}+\widehat{\boldsymbol{c}}_{m}
$$

In the presence of internal forces and torques $\mathcal{F}_{e}$, the controlled space robot is described in the following form, from (3) and (16):

$$
\ddot{\boldsymbol{x}}_{e}=\boldsymbol{y}+\boldsymbol{H}_{e}^{-1} \mathcal{F}_{e}
$$

that reveals the existence of a nonlinear coupling term due to the internal forces $\mathcal{F}_{e}$. Substituting (17) into (19) yields

$$
\boldsymbol{\Lambda}_{d} \ddot{\widetilde{\boldsymbol{x}}}_{e}+\boldsymbol{D}_{d} \dot{\widetilde{\boldsymbol{x}}}_{e}+\boldsymbol{K}_{d} \widetilde{\boldsymbol{x}}_{e}=\boldsymbol{\Lambda}_{d} \boldsymbol{H}_{e}^{-1} \mathcal{F}_{e}
$$

The expression in (20) establishes a relationship through a generalized mechanical impedance between the vector of resulting forces $\boldsymbol{\Lambda}_{b} \boldsymbol{H}_{e}^{-1} \mathcal{F}_{e}$ and the vector of displacements $\widetilde{\boldsymbol{x}}_{e}$ in the operational space.

To avoid the coupled motion attributed by $\boldsymbol{H}_{e}^{-1}$ in (20), it is necessary to measure the internal forces and torques 
between the target and the end-effector. In this case, the control law is selected as:

$$
\mathcal{F}_{i}=\boldsymbol{H}_{e} \boldsymbol{y}+\boldsymbol{c}_{e}-\mathcal{F}_{e}
$$

with

$$
\boldsymbol{y}=\ddot{\boldsymbol{x}}_{e}^{d}-\boldsymbol{\Lambda}_{d}^{-1}\left(\boldsymbol{D}_{d} \dot{\widetilde{\boldsymbol{x}}}_{e}+\boldsymbol{K}_{d} \widetilde{\boldsymbol{x}}_{e}\right) .
$$

The torque input can be expressed in function of the command $\mathcal{F}_{i}$ and of the internal forces $\mathcal{F}_{e}$ :

$$
\boldsymbol{\tau}=-\widehat{\boldsymbol{H}}_{m} \boldsymbol{H}_{e m}^{+} \mathcal{F}_{i}+\widehat{\boldsymbol{c}}_{m}+\boldsymbol{H}_{e m}^{T} \boldsymbol{H}_{e}^{-1} \mathcal{F}_{e} .
$$

On the assumption of no error on the force measurements, the following linear impedance can be achieved:

$$
\boldsymbol{\Lambda}_{d} \ddot{\widetilde{\boldsymbol{x}}}_{e}+\boldsymbol{D}_{d} \dot{\widetilde{\boldsymbol{x}}}_{e}+\boldsymbol{K}_{d} \widetilde{\boldsymbol{x}}_{e}=\mathbf{0} \text {. }
$$

With proper selection of the control gains, asymptotic stability is guaranteed. However, the component of the internal force $\mathcal{F}_{e}$ arising from the compound motion, never converges to zero, until the compound is itself stabilized in the inertial frame. As such, if the force is not measured, one can only expect that the resulting error remains bounded. Clearly, the magnitude of this residual error depends on the control parameters.

Furthermore, in case of force measurement, the external forces and torques can be compensated more easily, because of the resulting decoupling. In practice, the tolerance of the deviation error should be analyzed prior to the implementation.

\section{Simulation STUdY}

This section presents the numerical simulation results of a realistic three-dimensional model as shown in Fig. 1. The chaser robot has a 7 DOF manipulator mounted on the base satellite, whose dynamic parameters are shown in Table I. The size of the target is the same as that of the chaser satellite. It is assumed that prior to the contact, the target is tumbling around one axis at a constant angular velocity of $0.05[\mathrm{rad} / \mathrm{s}]$ at the capturing with the chaser-robot and that the end effector of the robot follows the grasping point on the target with the same velocity. The grasping point is deviated from the center of mass of the target by 0.5 [m]. As far as the end effector has the same velocity as that of the grasping point of the target, no impact between the two systems is induced.

In the simulation examples, the target parameters of the planned motion are given in Table II, while those of the controlled motion in Table I, giving the extent of uncertainty introduced in the system.

\section{A. Target uncertainty cases}

Two cases with different impedance characteristics are illustrated here. The values of inertia, damping and stiffness matrices on the end-effector are selected as shown in Table III and IV. To obtain the desired control performance one may also revert to several existing methods (e.g. Factorization design, Double diagonalization design) [12]. To set the parameters, care should be taken by considering how softly the end-effector grasps the target, to prevent it from bouncing away.

Figs. 4 to 6 illustrate the simulation results. Fig. 4 shows the velocity of the end-effector with respect to the base coordinate frame. Fig. 5 illustrates the motion of the base
TABLE I: Dynamic parameters for a space robot

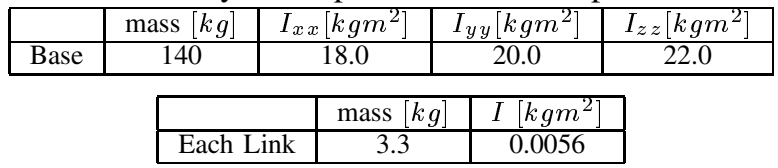

TABLE II: Estimated dynamic parameters for a target

\begin{tabular}{|c|c|c|c|c|}
\hline & mass $[\mathrm{kg}]$ & $I_{x x}\left[\mathrm{kgm}^{2}\right]$ & $I_{y y}\left[\mathrm{kgm}^{2}\right]$ & $I_{z z}\left[\mathrm{kgm}^{2}\right]$ \\
\hline Base & 50 & 10.0 & 10.0 & 10.0 \\
\hline
\end{tabular}

satellite. Fig. 6 shows the internal forces and torques due to the target. In these figures, the solid line depicts the desired trajectory, the dashed line depicts the case (1) and the dotted line depicts the case (2).

Even if parameter errors occur in the system, the endeffector finally converges to the desired trajectory with respect to the base coordinate frame. The base satellite continues to move in the inertial coordinate frame due to the non-zero momentum of the system. This motion can be compensated with extra actuators on the base, such as jet thrusters and reaction wheels. The compound motion in the inertial frame causes the bounded internal forces. However, the internal forces due to the target decrease to almost zero here. If the forces are measured, the error can be compensated properly.

Here it is clearly shown that the simple impedance control method is useful to follow the trajectory and we do not need any target parameters to implement the control.

\section{B. Initial impact case}

Furthermore, Fig. 7 shows the velocity profile of the endeffector in case of impact at the contact time $(t=0)$. The solid line depicts the desired trajectory, the dashed line depicts the case with impact, resulting in an initial velocity error of $0.05[\mathrm{rad} / \mathrm{s}]$. It is shown that the end-effector finally converges to the desired velocity with the same impedance control scheme.

\section{CONCLUSIONS}

A novel and very simple method is presented to derive a dynamic model for a free-floating robot in operational space, necessary for the desired control implementation. Furthermore, an impedance control theory is derived from this, based on feedback linearization, to account for target parameter uncertainty. However, the derived formulation of the control law is independent of the target parameters. The effectiveness of the method is shown with simulation results.

TABLE III: Desired impedance parameters - case (1)

\begin{tabular}{|c|c|c|c|c|c|c|}
\hline & $\mathrm{x}$ & $\mathrm{y}$ & $\mathrm{Z}$ & roll & pitch & yaw \\
\hline $\boldsymbol{\Lambda}_{d}$ & 100 & 100 & 100 & 300 & 300 & 300 \\
\hline $\boldsymbol{D}_{d}$ & 300 & 300 & 300 & 300 & 300 & 300 \\
\hline $\boldsymbol{K}_{d}$ & 100 & 100 & 100 & 500 & 500 & 500 \\
\hline
\end{tabular}

TABLE IV: Desired impedance parameters - case (2)

\begin{tabular}{|c|c|c|c|c|c|c|}
\hline & $\mathrm{x}$ & $\mathrm{y}$ & $\mathrm{Z}$ & roll & pitch & yaw \\
\hline $\boldsymbol{\Lambda}_{d}$ & 1.0 & 1.0 & 1.0 & 30 & 30 & 30 \\
\hline $\boldsymbol{D}_{d}$ & 0.05 & 0.05 & 0.05 & 20 & 20 & 20 \\
\hline $\boldsymbol{K}_{d}$ & 1.0 & 1.0 & 1.0 & 50 & 50 & 50 \\
\hline
\end{tabular}



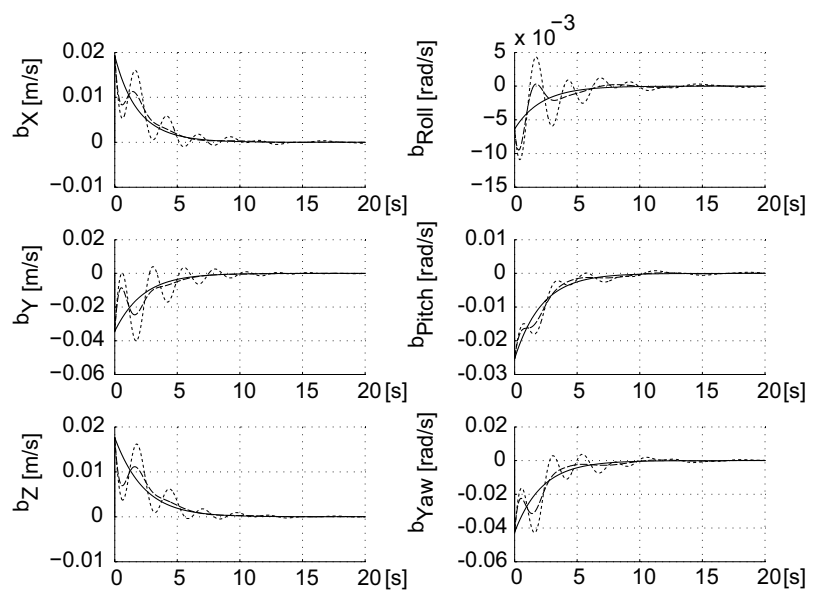

Fig. 4: Linear and angular velocity of the end-effector, ${ }^{b} \dot{\boldsymbol{x}}_{e}$
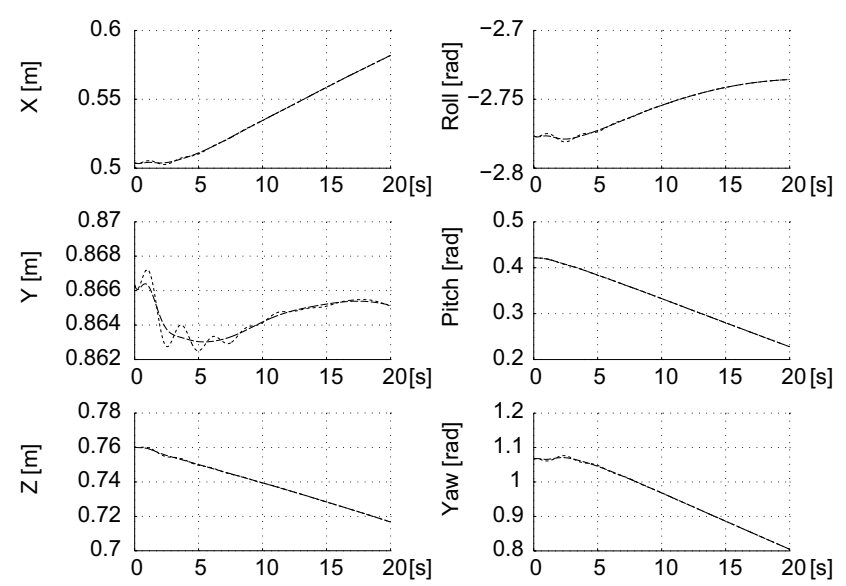

Fig. 5: Position and orientation of the base satellite, $\dot{x}_{b}$
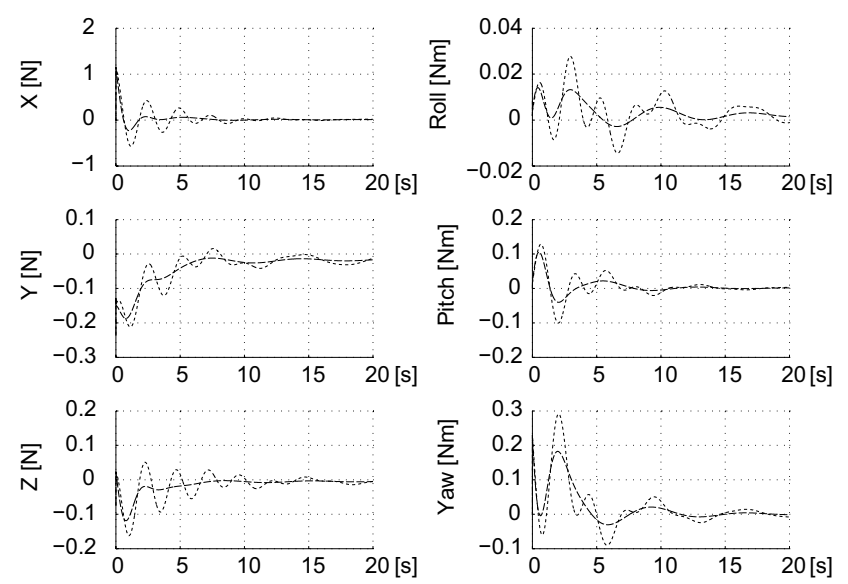

Fig. 6: Internal forces and torques due to the target, $\mathcal{F}_{e}$
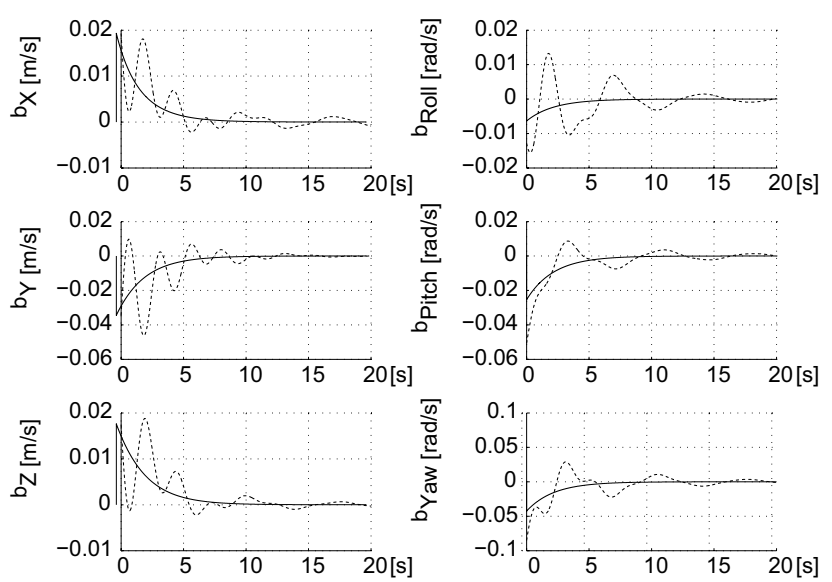

Fig. 7: Velocity of the end-effector in the initial impact case, ${ }^{b} \dot{\boldsymbol{x}}_{e}$

Finally, the developed method sets a base for more general compliance control tasks, which may include impact with the environment.

\section{REFERENCES}

[1] Y. Xu, H.-Y. Shum, J.-J. Lee, and T. Kanade, "Adaptive Control of Space Robot System with an Attitude Controlled Base," in Proc. of the 1992 Int. Conf. on Robotics and Automation, Nice, France, May 1992, pp. 2005 - 2011.

[2] Y. L. Gu and Y. Xu, "A Normal Form Augmentation Approach to Adaptive Control of Space Robot Systems," in Proc. of the 1993 IEEE Int. Conf. on Robotics and Automation, vol. 2, Atlanta, USA, May 1993, pp. $731-737$

[3] D. N. Dimitrov and K. Yoshida, "Momentum Distribution in a Space Manipulator for Facilitating the Post-Impact Control," in Proc. of the 2004 IEEE/RSJ Int. Conf. on Intelligent Robots and Systems, Sendai, Japan, September. 2004, pp. 3333 - 3338.

[4] D. N. Nenchev and K. Yoshida, "Impact Analysis and Post-Impact Motion Control Issues of a Free-Floating Space Robot Subject to a Force Impulse," IEEE Transactions on Robotics and Automation, vol. 15, no. 3, pp. 548 - 557, June 1999.

[5] K. Yoshida, R. Kurazume, N. Sashida, and Y. Umetani, "Modeling of Collision Dynamics for Space Free-Floating Links with Extended Generalized Inertia Tensor," in Proc. of the 1992 IEEE Int. Conf. on Robotics and Automation, Nice, France, May 1992, pp. 899 - 904.

[6] L.-B. Wee and M. W. Walker, "On the Dynamics of Contact between Space Robots and Configuration Control for Impact Minimization," IEEE Transactions on Robotics and Automation, vol. 9, no. 5, pp. 581 - 591, October 1993.

[7] Y. Xu and T. Kanade, Eds., Space Robotics: Dynamics and Control. Kluwer Academic Publishers, 1993.

[8] O. Khatib, "A Unified Approach for Motion and Force control of Robot Manipulators: The Operational Space Formulation," IEEE Journal of Robotics and Automation, vol. RA-3, no. 1, pp. 43 - 53, 1987.

[9] H. Asada, "A Geometrical Representation of Manipulator Dynamics and Its Application to Arm Design," Trans. ASME Journal Dynamic System Measurement Control, vol. 105, no. 3, pp. 131 - 135, 1983.

[10] L. Sciavicco and B. Siciliano, Modelling and Control of Robot Manipulators. Springer, 2000.

[11] K. Yoshida, H. Nakanishi, H. Ueno, N. Inaba, T. Nishimaki, and M. Oda, "Dynamics, Control and Impedance Matching for Robotic Capture of a Non-Cooperative Satellite," Advanced Robotics, vol. 18, no. 2, pp. 175 - 198, 2004.

[12] A. Albu-Schaffer, C. Ott, U. Frese, and G. Hirzinger, "Cartesian Impedance Control of Redundant Robots: Recent Results with the DLR-Light-Weight-Arms," in Proc. of the 2003 IEEE Int. Conf. of Robotics and Automation, Taipei, Taiwan, May 2003, pp. 3704 - 3709. 